\title{
Nearlattices Whose Sets of Principal $n$-ideals Form Relatively Normal Nearlattices
}

\author{
M. S. Raihan and A. S. A. Noor \\ Department of Mathematics, Rajshahi University, Rajshahi, Bangladesh \\ Department of APCE, East West University, Mohakhali, Dhaka, Bangladesh
}

Received 14 December 2009, accepted in final revised form 8 November 2010

\begin{abstract}
We generalize several results of relatively normal nearlattices in terms of $n$-ideals. We introduce the notion of relative $n$-annihilators in a nearlattice and include some interesting results on this. Several characterizations of the set of principal $n$-ideals $P_{n}(S)$ are given which forms a relatively normal nearlattice in terms of relative $n$-annihilators. It is shown that $P_{n}(S)$ is relatively normal if and only if for any two incomparable prime $n$-ideals $P$ and $Q, P \vee Q=\mathrm{L}$.
\end{abstract}

Keywords: Relatively normal nearlattice; Relative $n$-annihilator; Incomparable prime $n$ ideals.

(C) 2011 JSR Publications. ISSN: 2070-0237 (Print); 2070-0245 (Online). All rights reserved.

doi:10.3329/jsr.v3i1.3955

J. Sci. Res. 3 (1), 35-42 (2011)

\section{Introduction}

Relative annihilators in lattices and semi-lattices have been studied by many authors including Mandelker [1] and Varlet [2]. Cornish [3] has used the annihilators in studying relative normal lattices. On the other hand, relative annihilators in nearlattices have been studied by Noor and Islam [4]. Recently Noor and Ali [5] have studied the relative $n$ annihilators in a lattice $L$ for a fixed element $n \in L$

In this paper we have introduced the notion of relative $n$-annihilators in a nearlattice. Then with the help of relative $n$-annihilators we have studied those $P_{n}(S)$ which are relatively normal.

\section{Preliminaries}

A nearlattice is a meet semi lattice together with the property that any two elements possessing a common upper bound, have a supremum. A nearlattice $S$ is distributive if $x \wedge(y \vee z)=(x \wedge y) \vee(x \wedge z)$ for all $x, y, z \in S$ provided $y \vee z$ exists. 
For a fixed element $n \in S$, a convex sub nearlattice containing $n$ is called an $n$-ideal. The concept of $n$-ideals is a kind of generalization of ideals and filters of a nearlattice. Details on nearlattices and n-ideals in both lattices and nearlattices can be found in refs. [6-9].

An element $n$ of a nearlattice $S$ is called a standard element if for all $t, x, y \in S$

$t \wedge((x \wedge y) \vee(x \wedge n))=(t \wedge x \wedge y) \vee(t \wedge x \wedge n)$

Element $n$ is called neutral if

i) it is standard and

ii) $n \wedge((t \wedge x) \vee(t \wedge y))=(n \wedge t \wedge x) \vee(n \wedge t \wedge y)$ for all $t, x, y \in S$.

An element $n$ of a nearlattice $S$ is called a medial element if $m(x, n, y)=(x \wedge y) \vee(x \wedge n) \vee(y \wedge n)$ exists for all $x, y \in S$.

Element $n$ is called an upper element of $S$ if $x \vee n$ exists for every $x \in S$ Of course, every upper element is medial.

An element $n$ of a nearlattice $S$ is called a central element if it is upper, neutral and complemented in each interval containing it.

For a medial element $n$, an $n$-ideal $P$ of a nearlattice $S$ is called a prime $n$-ideal if $P \neq$ $S$ and $\mathrm{m}(x, n, y) \in P(x, y \in S)$ implies either $x \in P$ or $y \in P$.

The set of all $n$-ideals of a nearlattice $S$ is denoted by $I_{n}(S)$ which is an algebraic lattice. For two $n$-ideals $I$ and $J$ of a nearlattice $S$, the set theoretic intersection is their infimum. Moreover, when $n$ is standard and medial, then $I \cap J=\{\mathrm{m}(i, n, j): i \in I, j \in J\}$. According to [7], the supremum is defined by $I \vee J=\left\{x: i \wedge j \leq x \leq i_{1} \vee j_{1}\right\}$, for some $i, i_{1} \in I$ and $j, j_{1} \in J$ provided $i_{i_{1} \vee j_{1}}$ exists.

An $n$-ideal generated by a finite number of elements $a_{1}, a_{2}, \ldots, a_{\mathrm{m}}$ is called a finitely generated $n$-ideal, denoted by $\left.<a_{1}, a_{2}, \ldots, a_{\mathrm{m}}\right\rangle_{n}$. Following [8],

$<a_{1}, a_{2}, \ldots, a_{\mathrm{m}}>_{n}=\left\{y \in S: a_{1} \wedge \ldots \wedge a_{\mathrm{m}} \wedge n \leq y=\left(y \wedge a_{1}\right) \vee \ldots \vee\left(y \wedge a_{\mathrm{m}}\right) \vee(y \wedge n)\right\}$,

provided $S$ is distributive.

When $S$ is a lattice, $<a_{1}, a_{2}, \ldots ., a_{m}>_{n}$ is the interval $\left[a_{1} \wedge \ldots . \wedge a_{m} \wedge n, a_{1} \vee \ldots . \vee a_{m} \vee n\right]$.

The set of finitely generated $n$-ideals is denoted by $F_{n}(S)$ which is again a nearlattice. An $n$-ideal generated by a single element $a$ is called a principal $n$-ideal, denoted by $\langle a\rangle_{n}$. The set of principal $n$-ideals is denoted by $P_{n}(S)$.

By [8] we know that $<a>_{n} \cap<b>_{n}=<m(a, n, b)>_{n}$ when $n$ is standard and medial.

Thus $P_{n}(S)$ is a semi lattice when $n$ is medial and standard. Moreover by [8] it is a nearlattice if $n$ is neutral and upper.

Let $S$ be a nearlattice. For $a, b \in \mathrm{S},\langle a, b\rangle=\{x \in \mathrm{S}: x \wedge a \leq b\}$ is called the annihilator of a relative to $b$, or simply a relative annihilator. It is easy to see that in presence of distributivity, $<a, b>$ is an ideal of $S$. 
Also note that $\langle a, b\rangle=\langle a, a \wedge b\rangle$. Again for $a, b \in L$, where $L$ is a lattice we define $<a, b>_{\mathrm{d}}=\{x \in L: x \vee a \geq b\}$, which we call a dual annihilator of a relative to $\mathrm{b}$ or simply a dual relative annihilator. In presence of distributivity of $L,\langle a, b\rangle_{\mathrm{d}}$ is a dual ideal (filter).

For $a, b \in S$ and an upper element $n \in S$, we define,

$$
\begin{aligned}
<a, b>^{n} & =\left\{x \in S: m(a, n, x) \in<b>_{n}\right\} \\
& =\{x \in S: b \wedge n \leq \mathrm{m}(a, n, x) \leq b \vee n\} .
\end{aligned}
$$

We call $\langle a, b\rangle^{n}$ the annihilator of a relative to $b$ around the element $n$ or simply a relative n-annihilator. It is easy to see that for all $a, b \in S,<a, b>^{n}$ is always a convex subset containing $n$. In presence of distributivity, it can easily be seen that $\langle a, b\rangle^{n}$ is an $n$-ideal. If $0 \in S$, then putting $n=0$, we have, $\langle a, b\rangle^{n}=\langle a, b\rangle$.

For two $n$-ideals $A$ and $B$ of a nearlattice $S$,

$<A, B>$ denotes $\{x \in S: m(a, n, x) \in B$ for all $a \in A\}$, when $n$ is a medial element.

In presence of distributivity, clearly $<A, B>$ is an $n$-ideal. Moreover, we can easily show that $\langle a, b\rangle^{n}=\left\langle\langle a\rangle_{n},\langle b\rangle_{n}\right\rangle$.

A prime $n$-ideal $P$ of a nearlattice $S$ is called a minimal prime $n$-ideal if there exists no prime $n$-ideal $Q$ such that $Q \neq P$ and $Q \subseteq P$.

A distributive nearlattice $S$ with 0 is normal if every prime ideal of $S$ contains a unique minimal prime ideal. A distributive nearlattice $S$ is relatively normal if each interval $[x, y]$ in $S(x, y \in S) x<y$, is normal.

We start the paper with the following result on $n$-ideals due to [8].

Lemma 1.1 For a central elemenl $n \in S, P_{n}(S) \cong(n]^{\mathrm{d}} \times[n)$.

Following result is also essential for the development of this paper, which is due to [10].

Lemma 1.2 Let $S$ be a distributive near-lattice with an upper element $n$ and let $I, J$ be two n-ideals of S. Then for any $x \in I \vee J, x \vee n=i \vee j$ and $x \wedge n=i^{\prime} \wedge j^{\prime}$ for some $i, i^{\prime} \in$ $I, j, j^{\prime} \in J$ with $i, j \geq n$ and $i^{\prime}, j^{\prime} \leq n$.

Following result in lattices is due to [5] and can be proved by similar technique in case of nearlattices. This is also a generalization of Lemma 3.6 [3].

Theorem 1.3 Let $S$ be a distributive nearlattice with an upper element $n$. Then the following conditions hold.

(i) $\left.\left.<\langle x\rangle_{n} \vee<y\right\rangle_{n},\langle x\rangle_{n}\right\rangle=\left\langle\langle y\rangle_{n},\langle x\rangle_{n}\right\rangle$.

(ii) $\left\langle\langle x\rangle_{n}, J\right\rangle=\vee y \in J\left|\langle x\rangle_{n},\langle y\rangle_{n}\right\rangle$, the supremum of $n$-ideals $\left\langle\langle x\rangle_{n}\right.$, $\left.\langle y\rangle_{n}\right\rangle$ in the lattice of $n$-ideals of $S$, for any $x \in S$ and any $n$-ideal $J$.

Lemma 1.4 and lemma 1.5 are due to [5]. We prefer to omit the proofs as they are easy to prove. 
Lemma 1.4 Let $S$ be a distributive nearlattice with an upper element $n$. Suppose $a, b$, $c \in \mathrm{S}$.

(i) If $a, b, c \geq n$, then $\left\langle\langle m(a, n, b)\rangle_{n},\langle c\rangle_{n}\right\rangle=\left\langle\langle a\rangle_{n},\langle c\rangle_{n}\right\rangle$ $\vee\left\langle\langle b\rangle_{n},\langle c\rangle_{n}\right\rangle$ is equivalent to $\langle a \wedge b, c\rangle=\langle a, c\rangle \vee\langle b, c\rangle$.

(ii) If $a, b, c \leq n$, then $\left.<<m(a, n, b)>_{n},\langle c\rangle_{n}\right\rangle=\left\langle\langle a\rangle_{n},\langle c\rangle_{n}\right\rangle \vee\left\langle\langle b\rangle_{n}\right.$, $\left.\langle c\rangle_{n}\right\rangle$ is equivalent to $\langle a \vee b, c\rangle_{\mathrm{d}}=\langle a, c\rangle_{\mathrm{d}} \vee\langle b, c\rangle_{\mathrm{d} \cdot} \cdot$

Lemma 1.5 Let $S$ be a distributive nearlattice with an upper element $n$. Suppose $a, b$, $c \in \mathrm{S}$.

(i) If $a, b, c \geq n$ and $a \vee b$ exists, then $\left\langle\langle c\rangle_{n},\langle a\rangle_{n} \vee\langle b\rangle_{n}\right\rangle=\left\langle\langle c\rangle_{n},\langle a\rangle_{n}\right\rangle$ $\vee\left\langle\langle c\rangle_{n},\langle b\rangle_{n}\right\rangle$ is equivalent to $\langle c, a \vee b\rangle=\langle c, a\rangle \vee\langle c, b\rangle$.

(ii) If $a, b, c \leq n$, then $\left\langle\langle c\rangle_{n},\langle a\rangle_{n} \vee\langle b\rangle_{n}\right\rangle=\left\langle\langle c\rangle_{n},\langle a\rangle_{n}\right\rangle \vee\left\langle\langle c\rangle_{n}\right.$, $\left\langle b>_{n}>\right.$ is equivalent to $\langle c, a \wedge b\rangle_{\mathrm{d}}=\langle c, a\rangle_{\mathrm{d}} \vee\langle c, b\rangle_{\mathrm{d}}$.

(iii) For each $x, y \in L,[x \vee y)^{* d}=[x)^{* d} \vee[y)^{* d}$.

(iv) If $x \vee y=1$, then $[x)^{* d} \vee[y)^{* d}=L$.

Following result is due to Theorem 2.4 [3]:

Theorem 1.6: For a distributive lattice with 0 , the following conditions are equivalent.

(i) Any two distinct minimal prime ideals are comaximal,

(ii) $L$ is normal,

(iii) For any $\mathrm{x}, \mathrm{y} \in \mathrm{L},(\mathrm{x} \wedge \mathrm{y}]^{*}=(\mathrm{x}]^{*} \vee(\mathrm{y}]^{*}$,

(iv) For any $x, y \in L$ with $x \wedge y=0$ implies $(x]^{*} \vee(y]^{*}=L$.

Moreover, when $L$ has a largest element 1 , then each of the above conditions is equivalent to" for any $x, y \in L, x \wedge y=0$ implies $x_{1}, y_{1} \in L$ such that $x \wedge x_{1}=y \wedge y_{1}=0$ and $x_{1} \vee y_{1}=1 ”$.

The following result is also due to Theorem 3.7 [3]:

Theorem 1.7. Let $L$ be a distributive lattice. Let $a, b, c$ be arbitrary elements and $A, B$ be arbitrary ideals. Then the following conditions are equivalent.

(i) $L$ is relatively normal.

(ii) $<a, b>\vee<b, a>=L$.

(iii) $\langle c, a \vee b\rangle=\langle c, a\rangle \vee\langle c, b\rangle$.

(iv) $<$ (c), $A \vee B>=<(c], A>\vee<(c], B>$.

(v) $\langle a \wedge b, c\rangle=\langle a, c\rangle \vee<b, c\rangle$.

The following result has been proved by [5] in case of lattices. The idea of dual relative annihilators in nearlattices is not always possible. Since $(n]$ is a sublattice of $S$ for each $n \in S$, we have: 
Theorem 1.8. Let $a, b, c \in(n]$ be arbitrary elements and A, B be arbitrary filters on (n]. Then the following conditions are equivalent.

(i) ( $n$ ] is relatively normal.

(ii) $<a, b>_{\mathrm{d}} \vee<b, a>_{\mathrm{d}}=(n]$.

(iii) $<c, a \wedge b>_{\mathrm{d}}=<c, a>_{\mathrm{d}} \vee<c, b>_{\mathrm{d}}$.

(iv) $<[c), A \vee B>_{\mathrm{d}}=<[c), A>_{\mathrm{d}} \vee<[c), B>_{\mathrm{d}}$.

(v) $\langle a \vee b, c\rangle_{\mathrm{d}}=\langle a, c\rangle_{\mathrm{d}} \vee\langle b, c\rangle_{\mathrm{d}}$.

Now we prove our main results of this paper, which are generalizations of Theorem 3.7 [3] and Theorem 5 [1].

Theorem 1.9. Let $n$ be a central element of a distributive nearlattice. Suppose A, B are two n-ideals of $S$. Then for all $a, b, c \in S$ the following conditions are equivalent.

(i) $P_{n}(S)$ is relatively normal.

(ii) $\left.\left.\left.\left.\left.<<a\rangle_{n},<b\right\rangle_{n}\right\rangle \vee<<b\right\rangle_{n},<a\right\rangle_{n}\right\rangle=S$.

(iii) $\left\langle\langle c\rangle_{n},\langle a\rangle_{n} \vee\langle b\rangle_{n}\right\rangle=\left\langle\langle c\rangle_{n},\langle a\rangle_{n}\right\rangle \vee\left\langle\langle c\rangle_{n},\langle b\rangle_{n}\right\rangle$, whenever $a \vee b$ exists.

(iv) $\left\langle\langle c\rangle_{n}, A \vee B\right\rangle=\left\langle\langle c\rangle_{n}, A\right\rangle \vee\left\langle\langle c\rangle_{n}, B\right\rangle$.

(v) $\left.<<m(a, n, b)>_{n},\langle c\rangle_{n}\right\rangle=\left\langle\langle a\rangle_{n},\left\langle c>_{n}\right\rangle \vee\left\langle\langle b\rangle_{n},\langle c\rangle_{n}\right\rangle\right.$.

Proof. (i) $\Rightarrow$ (ii). Let $z \in S$. Consider the interval $I=\left[\langle a\rangle_{n} \cap\langle b\rangle_{n} \cap\langle z\rangle_{n},\langle z\rangle_{n}\right]$ in $P_{n}(S)$. Then $\langle a\rangle_{n} \cap\langle b\rangle_{n} \cap\langle z\rangle_{n}$ is the smallest element of the interval $I$. By (i), I is normal. Then by Theorem 1.6, there exist principal $n$-ideals $\left\langle p>_{n},\langle q\rangle_{n} \in I\right.$ such that, $\langle a\rangle_{n} \cap\langle z\rangle_{n} \cap\langle p\rangle_{n}=\langle a\rangle_{n} \cap\langle b\rangle_{n} \cap\langle z\rangle_{n}=\langle b\rangle_{n} \cap\langle z\rangle_{n} \cap\langle q\rangle_{n}$ and $<z>_{n}=<p>_{n} \vee<q>_{n}$.

Now, $\langle a\rangle_{n} \cap\langle p\rangle_{n}=\langle a\rangle_{n} \cap\langle p\rangle_{n} \cap\langle z\rangle_{n}=\langle a\rangle_{n} \cap\langle b\rangle_{n} \cap\langle z\rangle_{n} \subseteq\langle b\rangle_{n}$ implies $\left.\langle p\rangle_{n} \subseteq<\langle a\rangle_{n},\langle b\rangle_{n}\right\rangle$.

Also, $\langle b\rangle_{n} \cap\langle q\rangle_{n}=\langle b\rangle_{n} \cap\langle z\rangle_{n} \cap\langle q\rangle_{n}=\langle a\rangle_{n} \cap\langle b\rangle_{n} \cap\langle z\rangle_{n} \subseteq\langle a\rangle_{n}$ implies $\left.\langle q\rangle_{n} \subseteq<\langle b\rangle_{n},\langle a\rangle_{n}\right\rangle$

Thus $\langle z\rangle_{n} \subseteq\left\langle\langle a\rangle_{n},\langle b\rangle_{n}\right\rangle \vee\left\langle\langle b\rangle_{n},\langle a\rangle_{n}\right\rangle$ and so $\left.z \in<\langle a\rangle_{n},\langle b\rangle_{n}\right\rangle_{\vee}$ $<<b>_{n},<a>_{n}>$.

Hence $\left.<<a\rangle_{n},\langle b\rangle_{n}\right\rangle \vee<<b>_{n},\left\langle a>_{n}\right\rangle=S$.

(ii) $\Rightarrow$ (iii). Suppose (ii) holds and $a \vee b$ exists. For (iii), R.H.S. $\subseteq$ L.H.S. is obvious. Now, let $z \in\left\langle\langle c\rangle_{n},\langle a\rangle_{n} \vee\langle b\rangle_{n}\right\rangle$. Then $z \vee n \in\left\langle\langle c\rangle_{n},\langle a\rangle_{n} \vee\langle b\rangle_{n}\right\rangle$ and $m(z \vee n, n, c) \in\left\langle a>_{n} \vee<b>_{n}\right.$.

That is, $m(z \vee n, n, c) \in[a \wedge b \wedge n, a \vee b \vee n]$. This implies $(z \vee n) \wedge(c \vee n) \leq a \vee b \vee n$. Now, by (ii), $z \vee n \in<\langle a\rangle_{n},\left\langle b>_{n}\right\rangle \vee\left\langle\left\langle b>_{n},\langle a\rangle_{n}>\right.\right.$. So by Lemma 1.2, $z \vee n=$ $r \vee t$ for some $r \in<\left\langle a>_{n},\left\langle b>_{n}>\right.\right.$ and $t \in<\left\langle b>_{n},\left\langle a>_{n}\right\rangle, r, t \geq n\right.$. Then $b \wedge n=m(r, n, a)=r \wedge(a \vee n) \leq b \vee n$. 
Hence, $r \wedge(c \vee n)=r \wedge(z \vee n) \wedge(c \vee n) \leq r \wedge(a \vee b \vee n)=(r \wedge(a \vee n)) \vee(r \wedge(b \vee$ $n)) \leq(b \vee n)$. This implies $r \in\left\langle\langle c\rangle_{n},\langle b\rangle_{n}\right\rangle$. Similarly, $t \in\left\langle\langle c\rangle_{n},\langle a\rangle_{n}\right\rangle$. Hence $z \vee n \in\left\langle\langle c\rangle_{n},\langle a\rangle_{n}\right\rangle \vee\left\langle\langle c\rangle_{n},\langle b\rangle_{n}\right\rangle$.

Again, $z \in\left\langle\langle c\rangle_{n},\langle a\rangle_{n} \vee\langle b\rangle_{n}\right\rangle$ implies $z \wedge n \in\left\langle\langle c\rangle_{n},\langle a\rangle_{n} \vee\langle b\rangle_{n}\right\rangle$. A dual calculation of the above shows, $\left.z \wedge n \in\left\langle<c>_{n},\langle a\rangle_{n}\right\rangle \vee\langle<c\rangle_{n},\langle b\rangle_{n}\right\rangle$. Thus by convexity, $z \in\left\langle\langle c\rangle_{n},\langle a\rangle_{n}\right\rangle \vee\left\langle\langle c\rangle_{n},\langle b\rangle_{n}\right\rangle$ and so L.H.S. $\subseteq$ R.H.S. Hence (iii) holds.

(iii) $\Rightarrow$ (iv). Suppose (iii) holds. In (iv), R.H.S. $\subseteq$ L.H.S. is obvious.

Now let $x \in\left\langle\langle c\rangle_{n}, A \vee B>\right.$. Then $x \vee n \in\left\langle\langle c\rangle_{n}, A \vee B>\right.$. Thus $\mathrm{m}(x \vee n, n, c) \in A \vee B$. Now $m(x \vee n, n, c)=(x \vee n) \wedge(n \vee c) \geq n$ implies $m(x \vee n, n, c) \in(A \vee B) \cap[n)$. Hence by Theorem 1.3(ii), $x \vee n \in<\left\langle c>_{n}\right.$, $(A \cap[n)) \vee(B \cap[n))>=\vee_{r \in(A \cap[n)) \vee(B \cap[n))}<<c$ $\left.>_{n},\langle r\rangle_{n}\right\rangle$. But by Lemma 1.2, $r \in(A \cap[n)) \vee(B \cap[n))$ implies $r=s \vee t$ for some $s \in A$, $t \in B$ and $s, t \geq n$. Then by (iii),

$$
\begin{aligned}
& \left\langle\langle c\rangle_{n},\langle r\rangle_{n}\right\rangle=\left\langle\langle c\rangle_{n},\langle s \vee t\rangle_{n}\right\rangle=\left\langle\langle c\rangle_{n},\langle s\rangle_{n} \vee\langle t\rangle_{n}\right\rangle \\
& =\left\langle\langle c\rangle_{n},\langle s\rangle_{n}\right\rangle \vee\left\langle\langle c\rangle_{n},\langle t\rangle_{n}\right\rangle \subseteq\left\langle\langle c\rangle_{n}, A\right\rangle \vee\left\langle\langle c\rangle_{n}, B\right\rangle
\end{aligned}
$$

Hence $x \vee n \in\left\langle\langle c\rangle_{n}, A\right\rangle \vee\left\langle\langle c\rangle_{n}, B\right\rangle$. Also $x \in\left\langle\langle c\rangle_{n}, A \vee B\right\rangle$ implies $x \wedge n \in<<c>_{n}, A \vee B>$.

Since $\mathrm{m}(x \wedge n, n, c)=(x \wedge n) \vee(x \wedge c) \leq n$, so $x \wedge n \in<\left\langle c>_{n},(A \vee B) \cap(n]>\right.$. Then, by Theorem 1.3(ii),

$$
x \wedge n \in<\left\langle c>_{n},(A \cap(n]) \vee(B \cap(n])>=\vee_{i \in(A \cap(n]) \vee(B \cap(n])}<\left\langle c>_{n},<i>_{n} .\right.\right.
$$

Again, using Lemma 1.2, we see that $i=p \wedge q$ where $p \in A, q \in B$ and $p, q \leq n$. Then by (iii),

$$
\begin{aligned}
& \left\langle\langle c\rangle_{n},\langle i\rangle_{n}\right\rangle=\left\langle\langle c\rangle_{n},\langle p \wedge q\rangle_{n}\right\rangle=\left\langle\langle c\rangle_{n},\langle p\rangle_{n} \vee\langle q\rangle_{n}\right\rangle \\
& =\left\langle\langle c\rangle_{n},\langle p\rangle_{n}\right\rangle \vee\left\langle\langle c\rangle_{n},\langle q\rangle_{n}\right\rangle \subseteq\left\langle\langle c\rangle_{n}, A\right\rangle \vee\left\langle\langle c\rangle_{n}, B\right\rangle
\end{aligned}
$$

Hence $x \wedge n \in\left\langle\langle c\rangle_{n}, A\right\rangle \vee\left\langle\langle c\rangle_{n}, B\right\rangle$. Therefore, by convexity, $x \in\left\langle\langle c\rangle_{n}, A\right\rangle$ $\vee\left\langle\langle c\rangle_{n}, B\right\rangle$ and so L.H.S. $\subseteq$ R.H.S. Thus (iv) holds.

(iv) $\Rightarrow$ (iii) is trivial.

(ii) $\Rightarrow$ (v). Suppose (ii) holds. In (v), R.H.S. $\subseteq$ L.H.S. is obvious.

Now let $z \in<<\mathrm{m}(a, n, b)>_{n},\left\langle c>_{n}>\right.$ which implies $\left.z \vee n \in<\langle\mathrm{m}(a, n, b)\rangle_{n},\langle c\rangle_{n}\right\rangle$. By (ii), $\left.\left.z \vee n \in<\langle a\rangle_{n},\langle b\rangle_{n}\right\rangle \vee<\langle b\rangle_{n},\langle a\rangle_{n}\right\rangle$. Then by Theorem 1.2, $\mathrm{z} \vee n=x \vee y$ for some $x \in\left\langle\langle a\rangle_{n},\langle b\rangle_{n}\right\rangle$ and $y \in\left\langle\langle b\rangle_{n},\langle a\rangle_{n}\right\rangle$ and $x, y \geq n$.

Thus, $\langle x\rangle_{n} \cap\langle a\rangle_{n} \subseteq\langle b\rangle_{n}$ and so $\langle x\rangle_{n} \cap\langle a\rangle_{n}=\langle x\rangle_{n} \cap\langle a\rangle_{n} \cap\langle b\rangle_{n} \subseteq<z$ $\vee n>_{n} \cap\langle a\rangle_{n} \cap\langle b\rangle_{n}=\left\langle z \vee n>_{n} \cap<\mathrm{m}(a, n, b)\right\rangle_{n} \subseteq<c>_{n}$. This implies $x \in<<a$ $>_{n},<c>_{n}>$.

Similarly, $y \in\left\langle\langle b\rangle_{n},\langle c\rangle_{n}\right\rangle$ and so $z \vee n \in\left\langle\langle a\rangle_{n},\langle c\rangle_{n}\right\rangle \vee\left\langle\langle b\rangle_{n},\langle c\rangle_{n}\right\rangle$.

Similarly, a dual calculation of above shows that $z \wedge n \in\left\langle\langle a\rangle_{n},\langle c\rangle_{n}\right\rangle \vee\left\langle\langle b\rangle_{n}\right.$, $\left.\langle c\rangle_{n}\right\rangle$. Thus by convexity, $z \in\left\langle\langle a\rangle_{n},\langle c\rangle_{n}\right\rangle \vee\left\langle\langle b\rangle_{n},\langle c\rangle_{n}\right\rangle$ and so L.H.S. $\subseteq$ R.H.S. Hence (v) holds. 
(v) $\Rightarrow$ (i). Suppose (v) holds. Let $a, b, c \geq n$.

By (v), $\left\langle\langle m(a, n, b)\rangle_{n},\langle c\rangle_{n}\right\rangle=\left\langle\langle a\rangle_{n},\langle c\rangle_{n}\right\rangle \vee\left\langle\langle b\rangle_{n},\langle c\rangle_{n}\right\rangle$. But by Lemma 1.5(i), this is equivalent to $\langle a \wedge b, c\rangle=\langle a, c\rangle \vee\langle b, c\rangle$. Then by Theorem 1.7, this shows that $[n]$ is relatively normal. Similarly, for $a, b, c \leq n$, using Lemma 1.5(ii) and Theorem 1.8, we find that (n] is relatively normal. Therefore by Lemma $1.1, P_{\mathrm{n}}(S)$ is relatively normal.

Finally we need to prove that (iii) $\Rightarrow$ (i).

Suppose (iii) holds. Let $a, b, c \in S \cap\left[n\right.$ ). By (iii), $\left\langle\langle c\rangle_{n},\langle a\rangle_{n} \vee\langle b\rangle_{n}\right\rangle=\left\langle\langle c\rangle_{n}\right.$, $<$ $a\rangle_{n} \vee\left\langle\langle c\rangle_{n},\langle b\rangle_{n}\right.$, whenever $a \vee b$ exists. But by Lemma 1.6(i), this is equivalent to $\langle c, a \vee b\rangle=\langle c, a\rangle \vee\langle c, b\rangle$. Then by Theorem 1.7, this shows that [ $n$ ) is relatively normal.

Similarly, for $a, b, c \leq n$, using the Lemma 1.6(ii) and Theorem 1.8, we find that $(n]$ is relatively normal. Therefore by Lemma $1.1, P_{n}(S)$ is relatively normal.

We conclude the paper with the following result which is a generalization of a result in [11].

Theorem 1.10. Let $S$ be a distributive nearlattice. If $n$ is central in $S$, then the following conditions are equivalent.

(i) $P_{n}(S)$ is relatively normal.

(ii) Any two incomparable prime n-ideals $P$ and $Q, P \vee Q=S$.

Proof. (i) $\Rightarrow$ (ii). Suppose (i) holds. Let $P$ and $Q$ be two incomparable prime $n$-ideals of $S$. Then there exist $a, b \in \mathrm{S}$ such that $a \in P-Q$ and $b \in Q-P$.

Then $\langle a\rangle_{n} \subseteq P-Q$ and $\langle b\rangle_{n} \subseteq Q-P$. Since by (i), $\mathrm{P}_{n}(\mathrm{~S})$ is relatively normal, so by Theorem 1.9, $\left.\left.\left.<<a\rangle_{n},\langle b\rangle_{n}\right\rangle \vee<<b\right\rangle_{n},\langle a\rangle_{n}\right\rangle=S$.

But as $P, Q$ are prime, so it is easy to see that $\left\langle<a>_{n},\langle b\rangle_{n}\right\rangle \subseteq Q$ and $\left.<<b\right\rangle_{n}$, $<a\rangle_{n}>\subseteq P$.

Therefore, $S \subseteq P \vee Q$ and so $P \vee Q=S$. Thus (ii) holds.

(ii) $\Rightarrow$ (i). Suppose (ii) holds. Let $P_{1}$ and $Q_{1}$ be two incomparable prime ideals of $[n$ ). Then by [12], there exist two incomparable prime ideals $P$ and $Q$ of $S$ such that $P_{1}=P \cap[n)$ and $Q_{1}=Q \cap[n)$. Since $n \in P_{1}$ and $n \in Q_{1}$, so $P$ and $Q$ are in fact two incomparable prime $n$-ideals of $S$. Then by (ii), $P \vee Q=S$.

Therefore, $P_{1} \vee Q_{1}=(P \vee Q) \cap[n)=S \cap[n)=[n)$. Thus by [11], [n) is relatively normal.

Similarly, considering two prime filters of $(n]$ and proceeding as above and using the dual result of Theorem 3.5 [3] we find that $(n$ ] is relatively normal. Therefore, by Lemma $1.1, P_{n}(S)$ is relatively normal.

\section{References}

1. M. Mandelker, Duke Math. J. 40, 377 (1970). doi:10.1215/S0012-7094-70-03748-8

2. J. Varlet, Bull Austral. Math. Soc. 9, 169 (1973). doi:10.1017/S0004972700043094

3. W. H. Cornish, J. Austral. Math. Soc. 14, 200 (1972). doi:10.1017/S1446788700010041

4. A. S. A. Noor and M. A. K. S. Islam, The Rajshahi University Studies B 25, 117 (1997). 
5. A. S. A. Noor and M. A. Ali, The Rajshahi University Studies B 28, 141 (2000).

6. W. H. Cornish and A. S. A.Noor, Bull. Austral. Math. Soc. 26, 185 (1982). doi:10.1017/S0004972700005700

7. M. G. Hossain and A. S. A.Noor, The Rajshahi University Studies B 28, 105 (2000).

8. A. S. A. Noor and M. G. Hossain, The Rajshahi University Studies B 25, 187 (1997).

9. A. S. A. Noor and M. A. Latif, SEA Bull. Math. 22, 72 (1998).

10. M. S. Raihan and A. S. A.Noor, Nearlattices whose sets of principal $n$-ideals form normal Nearlattices - submitted (2010).

11. A. S. A. Noor, M. B. Rahman, and M. A. K. Azad, The Rajshahi University Studies B 32, 77 (2004).

12. M. B. Rahman and A. S. A. Noor, SEA Bull. Math. 23, 193 (1999). 